\title{
Giant peritoneal loose bodies
}

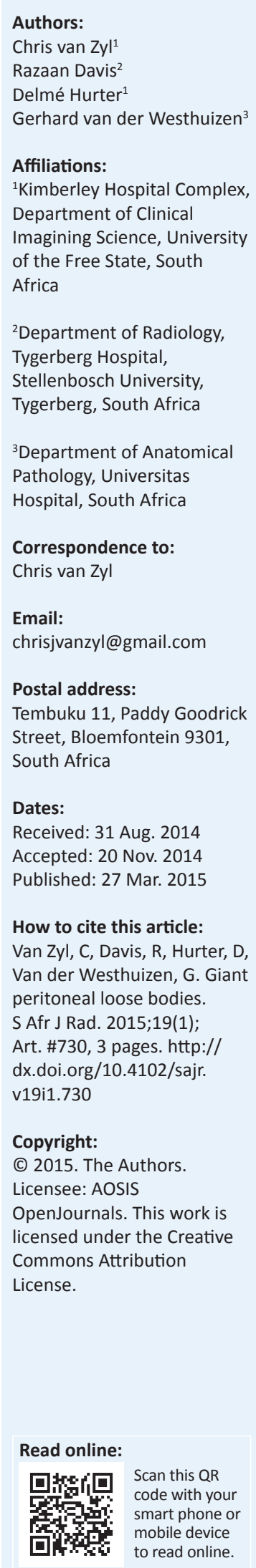

Giant peritoneal loose bodies are rare lesions, originating from auto-amputated appendices epiploicae. They may cause urinary or gastrointestinal obstruction and, should the radiologist not be familiar with the entity, can potentially be confused with malignant or parasitic lesions. Familiarity with their characteristic computed tomographic features is essential to prevent unnecessary surgery in the asymptomatic patient. We present a case of a 70-year-old man diagnosed with two giant peritoneal loose bodies.

\section{Introduction}

Giant peritoneal loose bodies, also known as peritoneal mice, ${ }^{1,2}$ are rare lesions with only a few reported cases in the literature. The recognition of these lesions is essential, as familiarity with their characteristic computed tomography (CT) features will assist with an accurate diagnosis, preventing unnecessary surgical intervention in an asymptomatic patient. ${ }^{3,4}$ It is important to differentiate giant peritoneal loose bodies from lesions such as gastrointestinal stromal tumour (GIST), fibroma, desmoid tumour, teratoma, mesenteric calcification, leomyoma, a retained surgical sponge and urinary, gallbladder and appendix stones. . $, 3,4,5,6,7^{-1}$

\section{Case presentation}

A 70-year-old man, with known hypertension, presented with a longstanding history of a tender enlarging abdominal mass in the left lower quadrant. His vital signs were normal and laboratory results were non-contributory.

Abdominal radiographs revealed well-circumscribed calcifications in the left upper and right lower quadrants. Subsequent abdominal ultrasound revealed what appeared to be aperistaltic thickened bowel loops containing large calcifications.

Two well-circumscribed soft-tissue masses were identified on abdominal CT, one just deep to the anterior abdominal wall at the level of the umbilicus, and the second in the left upper quadrant anterior to the left kidney. A tissue plane was clearly visible between these masses and the surrounding intra-abdominal structures. Each lesion had distinct alternating layers of density, namely an outer non-enhancing rim; a concentric layer of soft-tissue density; a hyperdense layer arranged in the form of petals; and, in the centre, a coarse lobulated calcification (Figure 1). A diagnosis of Echinococcus multilocularis was entertained, but the radiological findings were not typical and hydatid serology was negative.

During exploratory laparotomy, two mobile solid masses, measuring $65 \times 50 \times 35 \mathrm{~mm}$ and $95 \times$ $75 \times 55 \mathrm{~mm}$ respectively, were found adherent to the retroperitoneum and anterior abdominal wall. Macroscopically, both lesions were ovoid and very firm (Figure 2), matching the 'boiled egg' appearance described by Sewkani et al.; ${ }^{1}$ the cut surface revealed the alternating layers identified on CT.

Microscopic examination demonstrated dense acellular to paucicellular hyalinised connective tissue with a concentric arrangement around the central calcified areas (Figure 3). The hyalinised connective tissue demonstrated scattered small pinpoint calcifications, variable in size. There was no evidence of fat necrosis, dysplasia or malignancy. The central calcified areas were not examined histologically.

\section{Discussion}

Peritoneal loose bodies, also known as peritoneal mice, are mostly found as incidentalomas during laparotomy or autopsy. ${ }^{1,2,4}$ When small $(5 \mathrm{~mm}-25 \mathrm{~mm})$, these peritoneal loose bodies are asymptomatic; however, when they reach a diameter of $50 \mathrm{~mm}-100 \mathrm{~mm}$ ('giant' peritoneal loose 

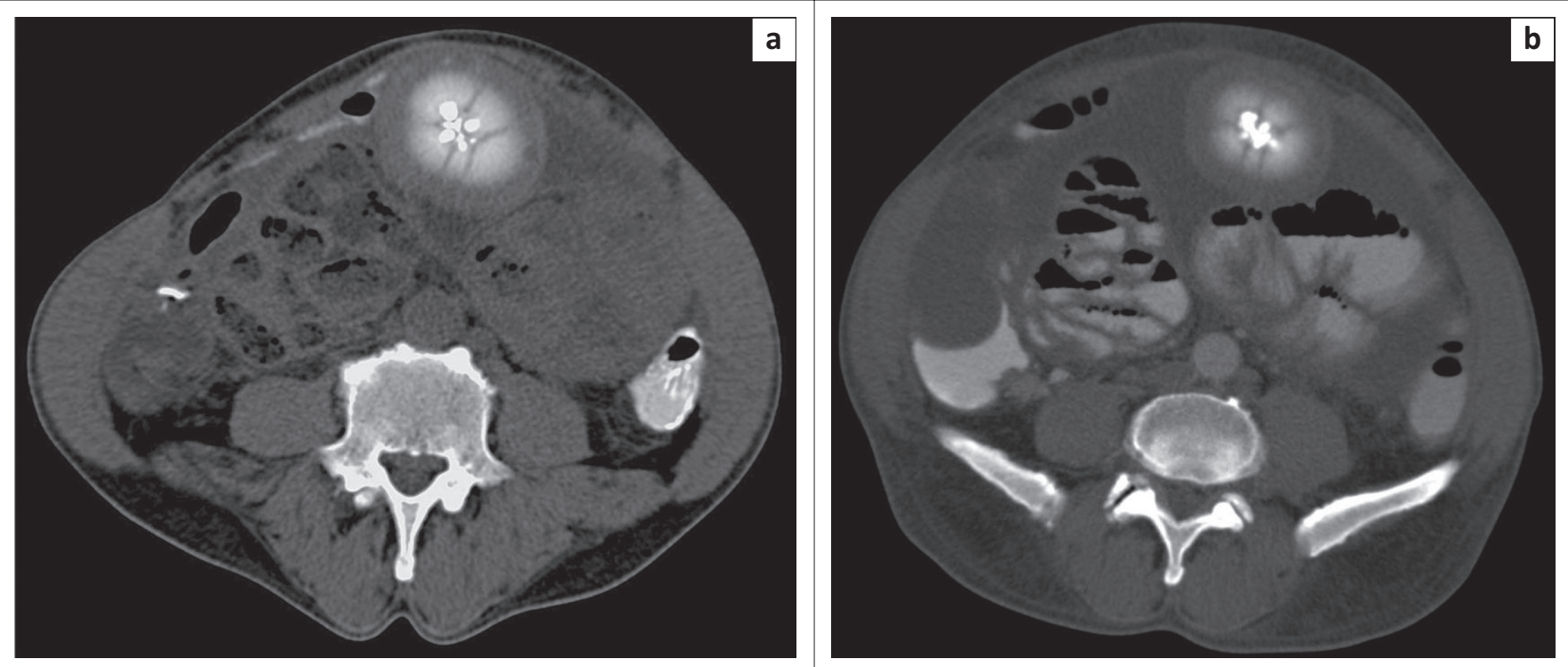

FIGURE 1: Pre- and post-contrast axial computed tomography (CT) images demonstrate the peritoneal 'mice' with alternating layers of density. Note the movement of the anterior mass between non-contrast-enhanced CT (a) and contrast-enhanced CT (b) acquisitions.
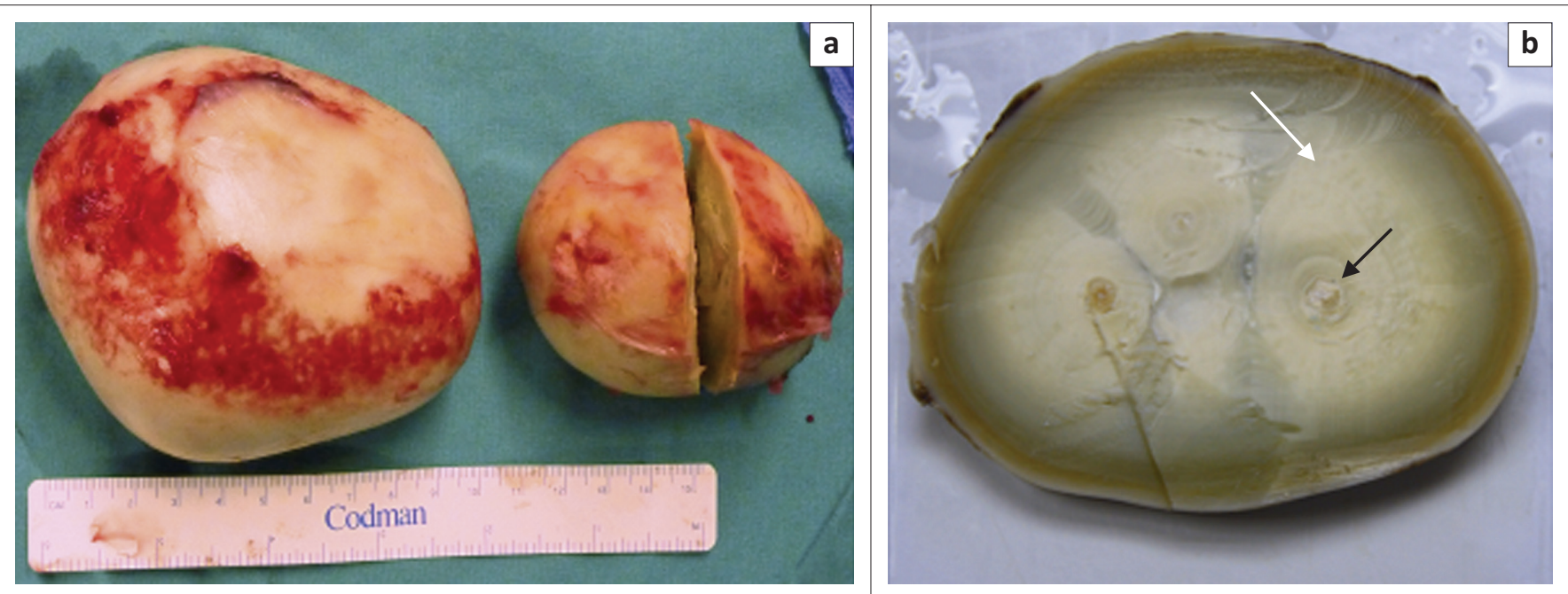

FIGURE 2: The two giant peritoneal loose bodies removed after laparotomy (a). Note the boiled egg appearance as described by Sewkani et al. ${ }^{1}$ A section (b) through one of the peritoneal loose bodies demonstrates the calcified centres (black arrow) with laminated fibrous connective tissue arranged concentrically around them (white arrow).

bodies or 'mice'), ${ }^{1,2}$ they may present with urinary retention and bowel obstruction, ${ }^{1,2}$ necessitating surgical removal.

Although the pathophysiology is still poorly understood, the current hypothesis, even as early as 1956, ${ }^{8}$ is that appendices epiploicae undergo chronic torsion, leading to saponification, calcification, fibrosis and, ultimately, auto amputation. Once free within the peritoneal cavity, exudative serum accumulates on the surface, leading to further growth. $1,2,3,4,5,6,7,8$ Peritoneal mice are usually found free within the peritoneal cavity but can re-attach (as with our patient), when they are known as parasitised peritoneal bodies. $^{1}$

Microscopically, these lesions consist of many layers of laminated fibrous tissue with a paucity of cellular elements, necrotic saponified fat, central calcification and peripheral proteinaceous material. ${ }^{1,2,6,7}$

The differential diagnoses for loose peritoneal bodies include gastrointestinal stromal tumour (GIST), fibroma, desmoid tumour, teratoma, mesenteric calcification, leiomyoma and urinary, gallbladder and appendix stones A retained sponge with a radio-opaque marker can also be mistaken for a loose peritoneal body; $;^{2,68}$ however, a retained surgical sponge is not likely to change position when the patient is rescanned in the prone position.

The CT features, including a well-defined, round or ovalshaped mass with central calcification, surrounded by nonenhancing peripheral soft tissue, are usually characteristic. ${ }^{3,4}$ A tissue plane between the lesion and surrounding structures 

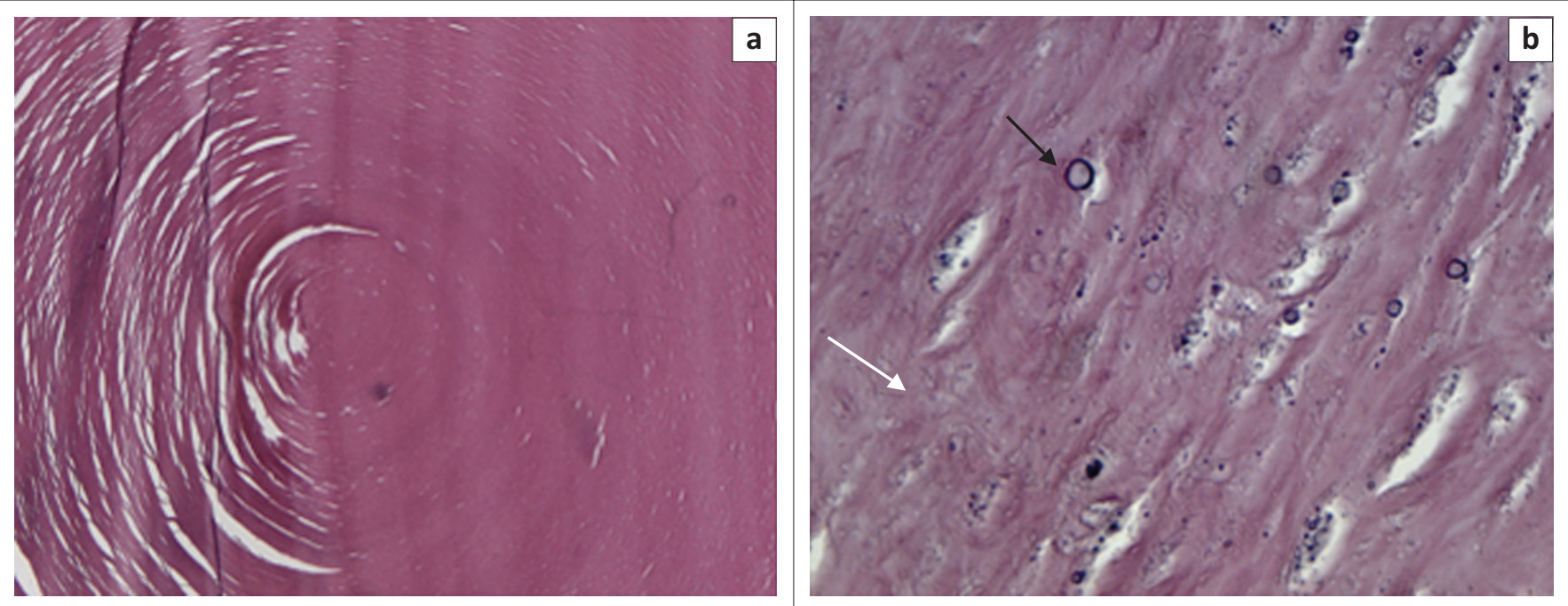

FIGURE 3: Concentric arrangement of hyalinised connective tissue (a). Punctate calcification (black arrow) in the paucicellular hyalinised fibrous connective tissue rings (white arrow) (b).

is usually seen. Variability in position also helps to confirm the diagnosis., ${ }^{2,3}$

\section{Conclusion}

The present case emphasises the importance of recognising the typical radiological appearance of this rare benign lesion, so preventing erroneous diagnosis of malignant or parasitic lesions and unnecessary surgery in an asymptomatic patient.

\section{Acknowledgements Competing interests}

The authors declare that they have no financial or personal relationship that may have inappropriately influenced them in writing this article.

\section{Authors' contributions}

C.v.Z. (University of the Free State) was the main author, with R.D. (Stellenbosch University) helping with the literature review and write-up. D.H. (University of the Free
State) also contributed to the initial write-up. G.v.d.W. (Universitas Hospital) was responsible for the histopathological evaluation.

\section{References}

1. Sewkani A, Jain A, Maudar K, et al. 'Boiled egg' in the peritoneal cavity - a giant peritoneal loose body in a 64-year-old man: A case report. J Med Case Rep. 2011;5:297. http://dx.doi.org/10.1186/1752-1947-5-297

2. Gayer G, Petrovitch I, Jeffrey RB. Foreign objects encountered in the abdominal cavity at CT. RadioGraphics. 2011;31:409-428. http://dx.doi.org/10.1148/rg.312105123

3. Gayer G, Petrovitch I. CT diagnosis of a large peritoneal loose body: A case report and review of the literature. Br J Radiol. 2011;84:e83-e85. http://dx.doi.org/10.1259/ bjr/98708052

4. Takayama S, Skamoto M, Takeyama H. Clinical challenges and images in GI. Image 1: Huge peritoneal loose body in the pelvic cavity. Gastroenterology. 2009;136:404. http://dx.doi.org/10.1053/j.gastro.2008.04.014

5. Takada A, Moriya Y, Muramatsu Y, et al. A case of giant peritoneal loose bodies mimicking calcified leiomyoma originating from the rectum. Jpn J Clin Oncol. 1998:28:441-442. http://dx.doi.org/10.1093/jjco/28.7.441

6. Takabe K, Greenberg JI, Blair SL. Giant peritoneal loose bodies. J Gastrointest Surg. 2006;10:465-468. http://dx.doi.org/10.1016/j.gassur.2005.05.003

7. Nomura $\mathrm{H}$, Hata $\mathrm{F}$, Yasoshima $\mathrm{T}$, et al. Giant peritoneal loose body in the pelvic cavity: Report of a case. Surg Today. 2003;33:791-793. http://dx.doi.org/10.1007/ s00595-003-2573-8

8. Southwood WF. Loose body in the peritoneal cavity. Lancet. 1956;271:1079. http://dx.doi.org/10.1016/S0140-6736(56)90211-2 